\title{
GENETIC ALGORITHM BASED DETECTION OF GENERAL
}

\section{LINEAR BICLUSTERS}

\author{
CUONG TO $^{1}$, ALAN WEE-CHUNG LIEW ${ }^{1}$ \\ ${ }^{1}$ School of Information and Communication Technology, Griffith University, Australia \\ E-MAIL: c.chieuto@griffith.edu.au, a.liew@griffith.edu.au
}

\begin{abstract}
:
Clustering methods classify patterns into clusters using the entire set of attributes of patterns in the similarity measurement. In plenty of cases, patterns are similar under a subset of attributes only. The class of methods that cluster patterns based on subsets of attributes is called biclustering. Biclustering simultaneously groups on both rows and columns of a data matrix and has been applied to various fields, especially gene expression data. However, the biclustering problem is inherently intractable and computationally complex. In recent years, several biclustering algorithms which are based on linear coherent model have been proposed. In this paper, we introduce a novel GA-based algorithm that uses hyperplane to describe the linear relationships between rows (genes) in a sub-matrix (bicluster). The performance of our algorithm is tested via simulated data, gene expression data and compared with several other bicluster methods.
\end{abstract}

\section{Keywords:}

Biclustering; Linear coherent patterns; Shifting and scaling patterns; Additive and multiplicative models; Gene expression data

\section{Introduction}

Microarrays are measurements of expression levels for thousands of genes in various biological conditions. The raw data of a microarray experiment is an image which is then converted into a numeric matrix called gene expression matrix. In a gene expression matrix, rows describe genes and columns are conditions. In order to extract biological knowledge from microarray data, plenty of machine learning methods have been proposed and one of the most popular problems is grouping genes according to their similarities, namely clustering.

Clustering is a technique that uses the entire set of attributes of patterns to classify patterns into clusters such that patterns belonging to the same cluster are similar and patterns of different clusters are dissimilar. But in several cases, a set of patterns are similar only on a subset of attributes, so the clustering algorithms do not have a good performance in such cases. In contrast, biclustering simultaneously clusters on both rows and columns of a data matrix. It has many applications to different fields such as text mining and information retrieval [3], economic data analysis [4, 5], biological data analysis [14, 16, 30], etc. A survey of biclustering can be found in [1, 2].

Several bicluster models have been proposed in the literature such as constant (values, rows, columns) model, additive model, multiplicative model, and linear model. For the constant model, all elements of a sub-matrix are a constant [6-8]. A fast divide-and-conquer approach [6] was used in the binary inclusion-maximal biclustering algorithm. Biclustering was considered as frequent itemset in DeBi [7]. QUBIC [8] solved the biclustering problem via finding a maximal and connected subgraph of weighted graph.

In the additive model, the values of a column are obtained by adding a constant value to other column [9, 10, 30]. In [9], hierarchical clustering and evolutionary algorithm are combined. The fitness function of evolutionary computation was defined as fraction of number of found biclusters, size of bicluster and mean square residue [10]. In [30], biclusters are exhaustively grown from seeds.

The multiplicative model means one column is a factor multiple of other columns [11-13]. In [11], Genetic algorithm was combined with greedy local search. Expectation maximization was applied to find parameters of probabilistic model in FABIA [12]. The objective function of SEBI [13] is the mean squared residue error.

In the linear model, the relationship between two columns is linear and it can be considered to be the general form of all the models mentioned above [14]. So far, the linear model has been the most sophisticated one and was used in the following algorithms [14-17], which proposed a geometric-based framework. Hough transform was applied directly to the high dimension feature spaces [14]. To tackle 
the high computation cost, Hough transform was performed in column-pair spaces using pairs of columns, and then various merging strategies are used to merge biclusters found in column spaces to form larger biclusters, such as hypergraph [15], additive and multiplicative pattern plot [16], and graph spectrum [17].

In this paper, we introduce a novel hyperplane based algorithm that combines evolutionary computation and gradient descent optimization method to find linear coherent patterns of biclusters. We introduce the basic concept and the proposed algorithm in Section 2. In Section 3, we present some experiment results and comparative studies. Finally, we draw some conclusion in Section 4.

\section{Method}

\subsection{Genetic algorithms (GAs)}

Genetic algorithms [20] are processes that mimic natural selection, evolution and adaptation of individuals in an environment. Consequently, the next generation is normally better than the previous one.

The scheme of genetic algorithms is that at the first step an initial population containing random individuals (solutions of problems) is created. Fitness value of each individual is then computed at the second step. At the third step, a new generation is created by using some operations such as reproduction, crossover, and mutation based on the fitness values. The second and third steps are looped until the criteria are satisfied. The criteria can be a maximum number of generations allowed to be run or an additional problem-specific success predicate which have to be satisfied. The solution of problem is the best-so-far individual (the best individual that ever appeared in any generation of the population).

In order to implement genetic algorithms, the following terms need to be determined: 1) fitness function creates a value for each individual and this value is used to estimate how good an individual is. Normally, the fitness function is the objective of the problem. 2) Individual is solution candidate of the problem. This step defines how to represent an individual. 3) Control parameters such as population size, number of generations and probabilities of crossover, mutation and reproduction are also determined.

\subsection{Biclustering models}

In the literature, several bicluster models have been proposed [1, 2] such as:

- Constant models (constant values, constant rows and constant columns): all elements of a bicluster (a row or a column) have the same value (Figure 1a, b, c).

- Additive model: the values of a column are obtained by adding a constant value to other column (Figure 1d).

- Multiplicative model: the values of a column are obtained by multiplying a constant value and other column (Figure 1e).

- Linear model: the relationship between two columns is linear (Figure 1f).

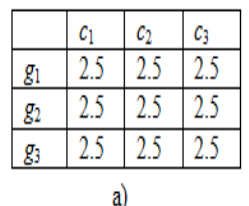

\begin{tabular}{|l|l|l|l|}
\hline & $c_{1}$ & $c_{2}$ & $c_{3}$ \\
\hline$g_{1}$ & 2.5 & 3.5 & 2.0 \\
\hline$g_{2}$ & 1.2 & 2.2 & 0.7 \\
\hline$g_{3}$ & 0.6 & 1.6 & 0.1 \\
\hline
\end{tabular}

d)

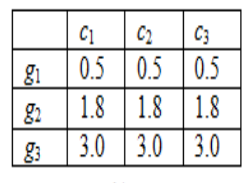

b)

\begin{tabular}{|l|l|l|l|}
\hline & $c_{1}$ & $c_{2}$ & $c_{3}$ \\
\hline$g_{1}$ & 2.5 & 5.0 & 3.75 \\
\hline$g_{2}$ & 1.2 & 2.4 & 1.8 \\
\hline$g_{3}$ & 0.6 & 1.2 & 0.9 \\
\hline
\end{tabular}

e)

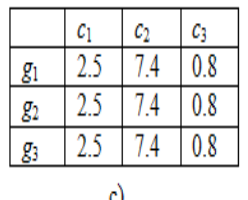

\begin{tabular}{|l|l|l|l|}
\hline & $c_{1}$ & $c_{2}$ & $c_{3}$ \\
\hline$g_{1}$ & 2.5 & 2.25 & 4.75 \\
\hline$g_{2}$ & 1.2 & 1.60 & 1.50 \\
\hline$g_{3}$ & 0.6 & 1.30 & 0.0 \\
\hline
\end{tabular}

\section{f)}

Figure 1. Illustration of various biclustering models: a) constant values, b) constant rows, c) constant columns, d) additive model, e) multiplicative model, f) linear model $\left(c_{2}=0.5 c_{1}+1, c_{3}=2.5 c_{1}-1.5\right)$

\subsection{The proposed algorithm}

Given an expression matrix, $\mathbf{A}(m \times n)$, where rows represent genes and columns describe conditions; a sub-matrix $(G, C) \subseteq \mathbf{A}$ where $G$ and $C$ are sets of genes (rows) and conditions (columns), respectively, is called a bicluster. The goal of our algorithm is to find biclusters which contain linear coherent patterns.

The proposed algorithm is based on the general linear model. In other words, we consider the coherence between patterns of a sub-matrix $(\mathrm{G}, \mathrm{C})$ formulated as a hyperplane [14]

$$
\sum_{i=1}^{|C|} u_{i} x_{i}=v
$$

where:

- $u_{1}, u_{2}, \ldots, u_{|\mathrm{C}|}, v \in \mathrm{R}$ and at least one of the $u_{i}$ is nonzero.

- $|\mathrm{C}|$ is the cardinality of the column set $C$.

The set of all points (genes) $\mathbf{x}=\left(x_{1}, x_{2}, \ldots, x_{|C|}\right)^{T}$ that satisfy the linear equation (1) is called a hyperplane of the space $\mathrm{R}^{|\mathrm{C}|}$. In the illustration (Figure 2), all genes $\left\{\mathbf{x}_{1}, \mathbf{x}_{2}, \mathbf{x}_{3}\right.$, $\left.\mathbf{x}_{4}, \mathbf{x}_{5}\right\}$ are on the same hyperplane whose equation is given by $c_{1}=0.5 c_{2}-1.5 c_{3}+2 c_{4}+3 c_{5}-c_{6}$. 


\begin{tabular}{rrrrrrr}
\hline & $\mathrm{C}_{1}$ & $\mathrm{C}_{2}$ & $\mathrm{C}_{3}$ & $\mathrm{C}_{4}$ & \multicolumn{1}{c}{$\mathrm{C}_{5}$} & \multicolumn{1}{c}{$\mathrm{c}_{6}$} \\
\hline $\mathbf{x}_{1}$ & 0.55 & 1 & 2.5 & 3 & 0.6 & 4 \\
$\mathbf{x}_{2}$ & 4.95 & 2.5 & 3 & 1.5 & 2 & 0.8 \\
$\mathbf{x}_{3}$ & 8.75 & 0.8 & 0.9 & 0.6 & 3 & 0.5 \\
$\mathbf{x}_{4}$ & 5.55 & 1.2 & 1.5 & 1.6 & 2.1 & 2.3 \\
$\mathbf{x}_{5}$ & 7.95 & 4.5 & 2.2 & 3.1 & 1.2 & 0.8 \\
\hline & & & & & &
\end{tabular}

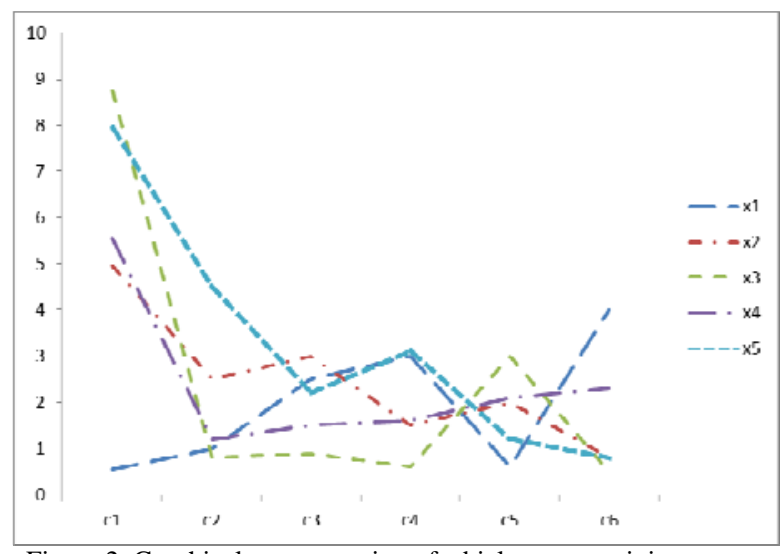

Figure 2. Graphical representation of a bicluster containing patterns whose relationship is $c_{1}=0.5 c_{2}-1.5 c_{3}+2 c_{4}+3 c_{5}-c_{6}$

Given the point (gene) $\mathbf{a}=\left(a_{1}, a_{2}, \ldots, a_{|C|}\right)^{\mathrm{T}}$ and $\mathrm{a}$ hyperplane $H=\left\{\mathbf{x} \in \mathrm{R}^{|\mathrm{C}|}: \mathbf{u}^{\mathrm{T}} \mathbf{x}=v\right\}$, the distance from $\mathbf{a}$ to $H$ is given by

$$
d(\mathbf{a}, H)=\frac{\left|\mathbf{u}^{\mathrm{T}} \mathbf{a}-v\right|}{\|\mathbf{u}\|}
$$

The proposed algorithm assumes that all genes of a bicluster (G, C) are on the same hyperplane (equation (1) hold) so the distances of genes in sub-matrix $(G, C)$ to the hyperplane $H$ must be zero. That is the perfect coherent patterns. In practice, we need to find the hyperplane that minimizes the total distances from the hyperplane to all genes. Therefore, the proposed algorithm for biclustering is stated as the following optimization problem:

Find $\mathbf{z}=\left(u_{1}, u_{2}, \ldots, u_{|C|}, v\right)^{\mathrm{T}}$ and a sub-matrix $(\mathrm{G}, \mathrm{C}) \subseteq \mathbf{A}$ which minimizes

$$
f(\mathbf{z}, \mathrm{G}, \mathrm{C})=\sum_{k=1}^{|\mathrm{G}|} d\left(\mathbf{x}_{k}, H\right)=\sum_{k=1}^{|\mathrm{G}|} \frac{\left|\mathbf{u}^{\mathrm{T}} \mathbf{x}_{k}-v\right|}{\|\mathbf{u}\|}
$$

Subject to:

$$
\left\{\begin{array}{c}
-1 \leq u_{i} \leq 1, i=1 . .|\mathrm{C}| \text {, and } \exists u_{i} \neq 0 \\
-1 \leq v \leq 1
\end{array}\right.
$$

In order to solve the above optimization problem, we need to find sub-matrix $(G, C)$ and parameters of hyperplane. This optimization problem is a mixture of discrete and continuous search spaces. Evolutionary computation is used to search for bicluster $(G, C)$ because the search space is discrete and evolutionary computation is well suited for this task [20]. To find the parameters of the hyperplane, we used the steepest descent method [21]. Finally, the whole scheme of the proposed algorithm is shown in Figure 3.

Step 1: Initialize a population of individuals which contain a set of biclusters $(\mathrm{G}, \mathrm{C})$.

Step 2: For each bicluster ( $G, C)$ of an individual, the steepest descent is used to obtain parameters of hyperplane via minimizing (3). The fitness value of each individual is computed using (5).

Step 3: Based on the fitness values, a new generation is created via reproduction, crossover and mutation operators.

Step 4: The $2^{\text {nd }}$ and $3^{\text {rd }}$ steps are iterated until maximum number of generation is achieved.

$$
\text { Figure 3. Outline of the proposed algorithm }
$$

Each individual which has a dynamic set of biclusters (G, C) is represented by integer string encoding. More specifically, each individual has two subsets containing selected rows and columns and separated by zero values. Figure 4 is an illustration of this encoding with three biclusters and the sets of rows and columns of the $1^{\text {st }}$ bicluster are $\{3,2,5\}$ and $\{1,8\}$, respectively.

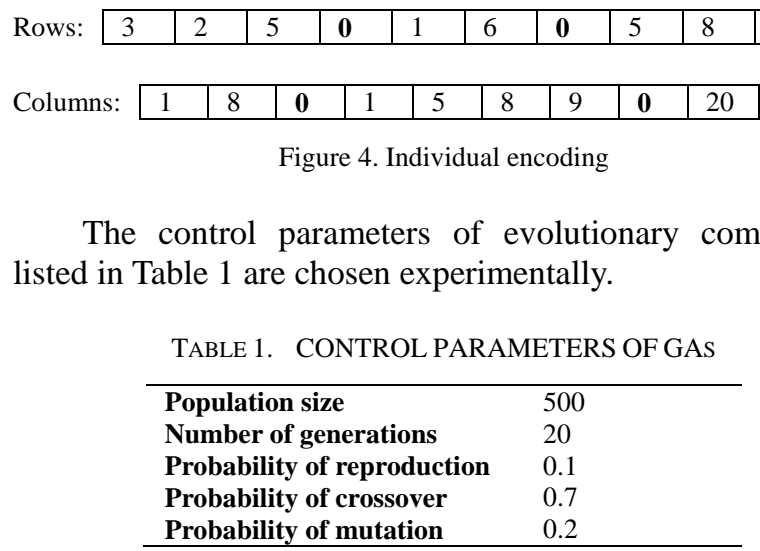

The fitness function of each individual is given by 


$$
\text { fitness }=\frac{\sum_{i=1}^{h} f_{i}(\mathbf{z}, \mathrm{G}, \mathrm{C})}{h}
$$

where $h$ is number of biclusters in an individual.

The iteration of the steepest descent method is stopped if the number of iterations reaches one hundred thousand or there are no changes in fitness function values between two consecutive iterations (e.g. $<1 \mathrm{e}-12$ ).

\section{Experiments}

In order to assess the performance of the proposed algorithm, we performed experiments on simulated data and real gene expression data. The simulated data were randomly generated with the number of biclusters known in advance. Moreover, the comparisons were also done with the following biclustering methods: FABIA [13], ISA 2 [22], xMOTIF [18], Cheng-Church [23], Spectral biclustering [19], Plaid Model [24].

\subsection{Simulated data}

One hundred independent datasets whose sizes are 200 rows by 40 columns were randomly created. For each dataset, there are four biclusters of general linear models and the number of rows and columns of biclusters were randomly selected within the ranges from 20 to 40 and between 7 and 15 , respectively.

The Jaccard coefficient that are used to measure the similarity between two biclusters is given by

$$
J\left(G_{1}, G_{2}\right)=\frac{\left|G_{1} \cap G_{2}\right|}{\left|G_{1} \cup G_{2}\right|}
$$

where $G_{1}$ and $G_{2}$ are two biclusters. The higher the Jaccard index, the better the performance of the algorithm.

The proposed algorithm and six other biclustering methods mentioned above were run on the 100 simulated datasets. The Jaccard indexes were computed from the results. Then, we computed the mean and standard deviation of the Jaccard index as listed in Table 2.

TABLE 2. MEAN AND STANDARD DEVIATION OF JACCARD INDEX BASED ON 100 SIMULATED DATASETS

\begin{tabular}{lll}
\hline Methods & Mean & Standard deviation \\
\hline The proposed algorithm & 0.4848 & 0.0264 \\
XMOTIF & N/A & N/A \\
Spectral biclustering & N/A & N/A \\
Plaid Model & 0.0329 & 0.1156 \\
Cheng-Church & 0.0333 & 0.0707 \\
ISA 2 & 0.1667 & 0.3430 \\
FABIA & 0.0036 & 0.0226 \\
\hline
\end{tabular}

Based on the results in Table 2, we see that the proposed algorithm gave the greatest mean and the smallest standard deviation. In other words, the proposed algorithm has the highest probability to find known biclusters and is the most stable method.

\subsection{Biological data}

We used gene expression dataset of diffuse large-B-cell lymphoma [25] in our validation. The diffuse large-B-cell lymphoma was used to predict the survival after chemotherapy and contains 180 samples of 661 genes.

In order to evaluate the bicluster results of the diffuse large-B-cell lymphoma, we verify them using Gene Ontology (GO) [26] and Kyoto Encyclopedia of Genes and Genomes (KEGG) pathway [27]. GO provides a dynamic, controlled vocabulary that can be applied to all eukaryotes and three ontologies are available: biological process, molecular function and cellular component [26]. Among the existing tools for GO and KEGG pathway, we selected GO-TermFinder [28] and ClueGO [29].

All biclusters obtained from biclustering algorithms were enriched to three functional GO categories such as GO biological process (GO BP), GO molecular function (GO MF), GO cellular component (GO CC), and the KEGG pathway. The biclusters that are found by the proposed algorithm and six selected biclustering methods were enriched by three GO categories and KEGG pathway shown in Tables 3-7. For this dataset, xMOTIF did not give any biclusters, and Cheng-Church considered whole database as a bicluster, while Spectral clustering and Plaid Model gave only one bicluster that was not significantly enriched by three GO categories and KEGG pathway.

TABLE 3. NUMBER OF BICLUSTERS FOUND BY SEVEN METHODS

\begin{tabular}{ll}
\hline Methods & Number of biclusters \\
\hline The proposed algorithm & 13 \\
XMOTIF & 0 \\
Spectral biclustering & 1 \\
Plaid Model & 1 \\
Cheng-Church & 1 \\
ISA 2 & 10 \\
FABIA & 5 \\
\hline
\end{tabular}

TABLE 4. NUMBER OF BICLUSTERS ENRICHED BY GO BP

\begin{tabular}{lll}
\hline Methods & p-value $=\mathbf{0 . 0 5}$ & p-value $=\mathbf{0 . 0 1}$ \\
\hline The proposed algorithm & 13 & 13 \\
XMOTIF & 0 & 0 \\
Spectral biclustering & 0 & 0 \\
Plaid Model & 0 & 0 \\
Cheng-Church & 1 & 1 \\
ISA 2 & 8 & 7 \\
FABIA & 5 & 5 \\
\hline
\end{tabular}


TABLE 5. NUMBER OF BICLUSTERS ENRICHED BY GO MF

\begin{tabular}{lll}
\hline Methods & p-value $=\mathbf{0 . 0 5}$ & p-value $=\mathbf{0 . 0 1}$ \\
\hline The proposed algorithm & 13 & 13 \\
xMOTIF & 0 & 0 \\
Spectral biclustering & 0 & 0 \\
Plaid Model & 0 & 0 \\
Cheng-Church & 1 & 1 \\
ISA 2 & 2 & 2 \\
FABIA & 3 & 3 \\
\hline
\end{tabular}

TABLE 6. NUMBER OF BICLUSTERS ENRICHED BY GO CC

\begin{tabular}{lll}
\hline Methods & $\boldsymbol{p}$-value $=\mathbf{0 . 0 5}$ & $\boldsymbol{p}$-value $=\mathbf{0 . 0 1}$ \\
\hline The proposed algorithm & 12 & 11 \\
xMOTIF & 0 & 0 \\
Spectral biclustering & 0 & 0 \\
Plaid Model & 0 & 0 \\
Cheng-Church & 1 & 1 \\
ISA 2 & 5 & 3 \\
FABIA & 3 & 1 \\
\hline
\end{tabular}

TABLE 7. NUMBER OF BICLUSTERS ENRICHED BY KEGG PATHWAY

\begin{tabular}{lll}
\hline Methods & p-value $=\mathbf{0 . 0 5}$ & p-value $=\mathbf{0 . 0 1}$ \\
\hline The proposed algorithm & 13 & 10 \\
XMOTIF & 0 & 0 \\
Spectral biclustering & 0 & 0 \\
Plaid Model & 0 & 0 \\
Cheng-Church & 1 & 1 \\
ISA 2 & 5 & 5 \\
FABIA & 4 & 3 \\
\hline
\end{tabular}

Based on the results obtained from the simulated datasets and gene expression data (Tables 2-7), the proposed algorithm has the best performance among the tested algorithms to find linear coherent biclusters and these biclusters are significantly enriched by three GO categories and KEGG pathway.

\section{Conclusions}

In this paper, we have presented a novel algorithm that can search linear coherent patterns in a dataset. The algorithm used genetic algorithms to search sub-matrices (biclusters) and for each sub-matrix, the steepest descent method determines the parameters of the hyperplane that has the minimum total distances between points (genes) and the hyperplane. The performance of the proposed algorithm was evaluated using simulated datasets and one real world microarray dataset, and was compared to several existing biclustering algorithms. The obtained biclusters found using the proposed algorithm were found to be significantly enriched biologically using three GO categories and KEGG pathway.

\section{References}

[1] Madeira, S.C., Oliveira, A.L.., "Biclustering algorithms for biological data analysis: a survey", IEEE Transactions on Computational Biology and Bioinformatics, 1, pp. 24-45, 2004.

[2] Zhao H., Liew A.W.C., Wang D.Z., Yan H., "Biclustering Analysis for Pattern Discovery: Current Techniques, Comparative Studies and Applications", Current Bioinformatics, 7(1), pp. 43-55, 2012..

[3] Dhillon, I.S., "Co-clustering documents and words using bipartite spectral graph partitioning", Proceeding of the seventh ACM SIGKDD international conference on Knowledge and discovery and data mining, pp. 269-274, 2001.

[4] Wang, H., Wang, W., Yang, J., Yu, P.S., “Clustering by pattern similarity in large data sets", Proceeding of the 2002 ACM SIGMOD international conference on Management of data, pp. 394-405, 2002.

[5] Yang, J., Wang, W., Wang, H., Yu, P., "delta-Clusters: capturing subspace correlation in a large data set", Proceeding of 18th IEEE international conference on Data Engineering, 2002.

[6] Li, G., Ma, Q., Tang, H., Paterson, A.H., Xu, Y., "QUBIC: a qualitative algorithm for analyses of gene expression data”, Nucleic Acids Research, 37, e101, 2009.

[7] Serin, A., Vingron, M., "DeBi: discovering differentially expressed biclusters using a frequent itemset approach", Algorithms for Molecular Biology, 6:18, 2011.

[8] Prelic, A., Bleuler, S., Zimmermann, P., Wille, A., Buhlmann, P., Gruissem, W., Hennig, L. Thiele, L., Zitzler, E., "A systematic comparison and evaluation of biclustering methods for gene expression data", Bioinformatics, 22, pp. 1122-1129, 2006.

[9] Huang, Q., Tao, D., Li, X., Liew, A.W.C., "Parallelized evolutionary learning for detection of biclusters in gene expression data”, IEEE/ACM Transactions on Computational Biology and Bioinformatics, 9, pp. 560-570, 2012.

[10] Huang, Q., Lu, M., Yan, H., “An evolutionary algorithm for discovering biclusters in gene expression data of breast cancer", IEEE Congress on Evolutionary Computation, pp. 829-834, 2008.

[11] Chakraborty, A., Maka, H., "Biclustering of gene expression data using genetic algorithm”, Proceeding of the IEEE Symposium on Computational Intelligence in Bioinformatics and Computational Biology, pp. 1-8, 2005.

[12] Hochreiter, S., Bodenhofer, U., Heusel, M., Mayr, A., Mitterecker, A., Kasim, A., Khamiakova, T., Van Sanden, S., Lin, D., Talloen, W., Bijnens, L., Göhlmann, H.W., Shkedy, Z., Clevert, D.A., "FABIA: factor analysis for bicluster acquisition”, Bioinformatics, 26, pp. 
1520-1527, 2010.

[13] Divina, F., Aguilar-Ruiz, J.S., "Biclustering of expression data with evolutionary computation”, IEEE Transactions on Knowledge and Data Engineering, 18, pp. 590-602, 2006.

[14] Gan, X., Liew, A.W.C., Yan, H., "Discovering biclusters in gene expression data based on high-dimensional linear geometries”, BMC Bioinformatics, 9: 209, 2008.

[15] Wang, Z. Yu, C.W., Cheung, R.C.C., Yan, H., "Hypergraph based geometric biclustering algorithm", Pattern Recognition Letters, 33, pp. 1656-1665, 2012.

[16] Zhao, H., Liew, A.W.C., Xie, X., Yan, H., “A new geometric biclustering algorithm based on the Hough transform for analysis of large-scale microarray data”, Journal of Theoretical Biology, 251, pp. 264-274, 2008.

[17] Wang, D.Z., Yan, H., "A graph spectrum based geometric biclustering algorithm”, Journal of Theoretical Biology, 317, pp. 200-211, 2013.

[18] Divina, F., Pontes, B., Giráldez, R., Aguilar-Ruiz, J.S., "An effective measure for assessing the quality of biclusters”, Computers in Biology and Medicine, 42, pp. 245-256, 2012.

[19] Bergmann, S., Ihmels, J., Barkai, N., "Iterative signature algorithm for the analysis of large-scale gene expression data”, Physical Review, E 67, 2003.

[20] Mitchell, M., An introduction to genetic algorithm, MIT Press, London, 2001.

[21] Hagan, M.T., Demuth, H.B., Beale, M.H., Neural network design, PWS Pub. Co, 1995.

[22] Ihmels, J., Bergmann, S., Barkai, N., "Defining transcription modules using large-scale gene expression data”, Bioinformatics, 20, pp. 1993-2003, 2004.
[23] Cheng, Y., Church, G.M., "Biclustering of expression data”, Proceeding of the Eighth International Conference on Intelligent Systems for Molecular Biology, pp. 93-103, 2000.

[24] Turner, H., Bailey, T., Krzanowski, W., "Improved biclustering of microarray data demonstrated through systematic performance tests" Computational Statistics and Data Analysis, 48, pp. 235-254, 2005.

[25] Rosenwald, A., et al. "The use of molecular profiling to predict survival after chemotherapy for diffuse large-B-cell lymphoma”, New Engl. J. Med., 346, pp. 1937-1947, 2002.

[26] Ashburner, M., Ball, C.A., et al., “Gene Ontology: tool for the unification of biology”, Nature Genetics, 25, pp. 25-29, 2000.

[27] Kanehisa, M., Goto, S., "KEGG: Kyoto Encyclopedia of Genes and Genomes”, Nucleic Acids Res., 28, pp. 27-30, 2000.

[28] Boyle, E.I., Weng, S., Gollub, J., Jin, H., Botstein, D., Cherry, J.M., Sherlock, G., "GO::TermFinder--open source software for accessing Gene Ontology information and finding significantly enriched Gene Ontology terms associated with a list of genes. Bioinformatics, 20, pp. 3710-3715, 2004.

[29] Bindea, G., Mlecnik, B., et al., "ClueGO: a Cytoscape plug-in to decipher functionally grouped gene ontology and pathway annotation networks”, Bioinformatics, 25, pp. 1091-1093, 2009.

[30] An J.Y., Liew A.W.C., Nelson C.C., "Seed-based biclustering of Gene Expression Data”, PLoS ONE, 7(8):e42431, 2012. 